\title{
Morfología Radicular y Mediciones Apicales en Primeros Molares en una Población Maya
}

\author{
Root Morphology and Apical Measurements of First Molars in a Mayan Population
}

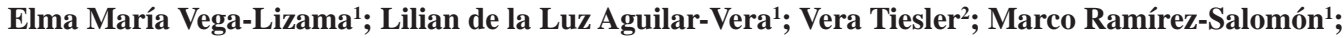 \\ Gabriel Alvarado-Cárdenas'; María Eugenia López-Villanueva1 \& Andrea Cucina ${ }^{1}$
}

\begin{abstract}
VEGA-LIZAMA, E. M.; AGUILAR-VERA, L. L.; TIESLER, V.; RAMÍREZ-SALOMÓN, M.; ALVARADO-CÁRDENAS, G.; LÓPEZ-VILLANUEVA, M. E. \& CUCINA, A. Morfología radicular y mediciones apicales en primeros molares en una población maya. Int. J. Morphol., 36(4):1229-1234, 2018.
\end{abstract}

RESUMEN: El conocimiento preciso de la morfología radicular dental son claves para el éxito en las terapias endodónticas. El objetivo de este estudio fue evaluar las distintas variaciones de la morfología radicular y apical en primeros molares superiores e inferiores de una población maya moderna. Se observaron microfotografías provenientes de 80 primeros molares superiores e inferiores, presentadas en cortes transversales a 1 y $2,5 \mathrm{~mm}$ del vértice apical, así como de las porciones ${ }^{1 / 3},{ }^{1 / 2},{ }^{2 / 3}$ del largo radicular y en la unión amelocementaria. Se determinó el número de raíces, conductos y configuración de Vertucci, así como forma y medidas de diámetro mayor y menor a 1 mm del vértice apical. El $90 \%$ de primeros molares superiores reportó la presencia de 3 raíces y el $10 \%$ mostró 2 . En tanto que el total de primeros molares inferiores (100\%) reportó 2 raíces. En general, en las raíces mesiales prevaleció el Tipo II de Vertucci mientras que en las distales y palatinas la Tipo I. En el corte a $1 \mathrm{~mm}$ del vértice apical la forma redonda fue la de mayor prevalencia en los conductos de primeros molares superiores $(44,5 \%)$, seguida de la irregular $(34,1 \%)$ y la oval $(21,4 \%)$; mientras que en los primeros molares inferiores la más prevalente fue la irregular $(54,5 \%)$, seguida de la redonda $(23,9 \%)$ y oval $(21,6 \%)$. Los diámetros mayor y menor de los conductos mesiales a $1 \mathrm{~mm}$ apical midieron 0,46 y $0,23 \mathrm{~mm}$ y 0,64 y $0,25 \mathrm{~mm}$ en molares superiores e inferiores respectivamente. La frecuencia de MB2 en primeros molares superiores fue del 77,8\%. Los conductos con mayor variabilidad fueron los mesiales. Se observó baja frecuencia de conductos ovales. Los diámetros mayores de los conductos mesiales en general, fueron superiores a $0,45 \mathrm{~mm}$.

PALABRAS CLAVE: Anatomía radicular; Conducto radicular; Primer molar; Diámetro apical.

\section{INTRODUCCIÓN}

El tratamiento endodóntico exitoso está basado en una secuencia clínica que incluye limpieza, desinfección, instrumentación biomecánica, obturación y sellado apical (Hargreaves \& Berman, 2016). Los primeros molares superiores son piezas con generalmente 3 raíces: una raíz mesiobucal (MB), una distobucal (DB), y una raíz palatina (P), y generalmente presentan 3 o 4 conductos (MB, DB, P, $2 \mathrm{MB}$ ). Por otro lado, el primer molar inferior típicamente presenta 2 raíces bien definidas, una mesial (M) caracterizada por dos conductos: mesiobucal (MB) y mesiolingual (ML), así como una raíz distal (D) con uno o dos conductos (de Pablo et al., 2010). El entendimiento de la anatomía radicular en ambas piezas es necesario antes de su tratamiento con el fin de elevar el pronóstico de éste. La región apical es considerada la zona crítica para la contaminación bacteriana. Una respuesta inmune ideal tendría lugar si la mayoría del tejido pulpar contaminado fuera eliminado de la región de la constricción apical, la cual corresponde anatómicamente al final del con- ducto con el menor diámetro y donde se encuentra la unión cemento-dentina (Abarca et al., 2014). La constricción apical (llamada por algunos autores forámen fisiológico) no siempre es identificable desde un punto de vista clínico o radiográfico. De esta forma, un límite convencional ha sido 1, 1,5 o incluso $2 \mathrm{~mm}$ del vértice apical (Gani \& Visvisian, 1999). Factores como la etnicidad, edad y factores locales contribuyen a las variaciones en la morfología radicular y apical (Oliva Rodríguez et al., 2017).

\section{MATERIAL Y MÉTODO}

Se examinaron 80 primeros molares permanentes extraídos (40 superiores y 40 inferiores) provenientes de pacientes yucatecos de origen maya entre 18 y 90 años. Todas las piezas presentaron formación radicular completa y

${ }^{1}$ Facultad de Odontología, Universidad Autónoma de Yucatán. Calle 61a No. 492a por Av. Itzáes. C.P. 97000, Mérida, Yucatán, México.

${ }^{2}$ Facultad de Ciencias Antropológicas, Universidad Autónoma de Yucatán. Km 1 Carretera Mérida-Tizimín C.P. 97305, Mérida, Yucatán, México. 
los motivos de extracción fueron caries no tratable y/o enfermedad periodontal grave. Los molares fueron desinfectados con hipoclorito de sodio al $2,5 \%$ y ultrasonido (NSK Varios 560 ®) y posteriormente fueron marcados por la cara bucal. Finalmente se colocaron en bolsas individuales con un número de folio de identificación.

Análisis de secciones transversales. Cada diente se midió, se fijó y se seccionó perpendicularmente al eje de la raíz en seis secciones para permitir la inspección de la morfología del conducto radicular. Se obtuvieron secciones transversales de $1 \mathrm{~mm}$ y $2,5 \mathrm{~mm}$ desde el extremo apical, así como $1 /$ $3,1 / 2,2 / 3$ de la longitud de la raíz y de la unión cementoesmalte; utilizando un disco de diamante de $0,5 \mathrm{~mm}$ (IsoMet 20LC, $5 \circledR^{\circledR}$ Alemania Diamond Wafering Blade) bajo agua corriente (Buehler IsoMet 1000 ® Germany). Cada sección se secó y se colocó en portaobjetos. Posteriormente se obtuvieron microfotografías digitales calibradas a $35 \mathrm{X}$ mediante el uso del microscopio estereoscópico Leica EZ4D ${ }^{\circledR}$ utilizando el software LAS ES ®. Una vez obtenidas todas las imágenes se procedió a determinar el número de raíces y conductos de cada pieza, así como la configuración anatómica y las mediciones apicales (diámetros mayor y menor conductos radiculares) a $1 \mathrm{~mm}$ del vértice apical. Se determinaron tres formas del conducto radicular: redondo, ovalado e irregular. Se consideró redondo al conducto con forma esférica o circular en el que los diámetros fueran similares, ovalado al conducto en el que el diámetro mayor fuera igual o mayor a 1,5 veces el diámetro menor, y conducto irregular a aquél que no cumplió con la definición de redondo u ovalado. Para las mediciones apicales se consideró diámetro mayor (DM) a la medida de mayor longitud entre dos puntos opuestos del perímetro del conducto que pasara por el centro de éste, mientras que diámetro menor $(\mathrm{Dm})$ fue la medida de menor longitud entre dos puntos opuestos del perímetro y que pasara por el centro del conducto. Las características cualitativas y cuantitativas de los conductos radiculares se observaron y registraron en una base de datos Excel ${ }^{\circledR}$. Posteriormente se organizaron los resultados en tablas y se calcularon las frecuencias, promedios y desviaciones estándar.

\section{RESULTADOS}

Los 36 primeros molares superiores (90\%) tuvieron 3 raíces (MB, DB y P) y 4 (10\%) tuvieron 2 . El $17,5 \%$ de primeros molares superiores reportó 3 conductos desde el corte cervical hasta el corte a $1 \mathrm{~mm}$ del vértice apical, independientemente del número de raíces; mientras que el 82,5 $\%$ de los casos mostraron variaciones en número y trayectoria de conductos radiculares (Tabla I).

Según la clasificación de Vertucci, el sistema de conductos radiculares más común en las raíces mesiobucales

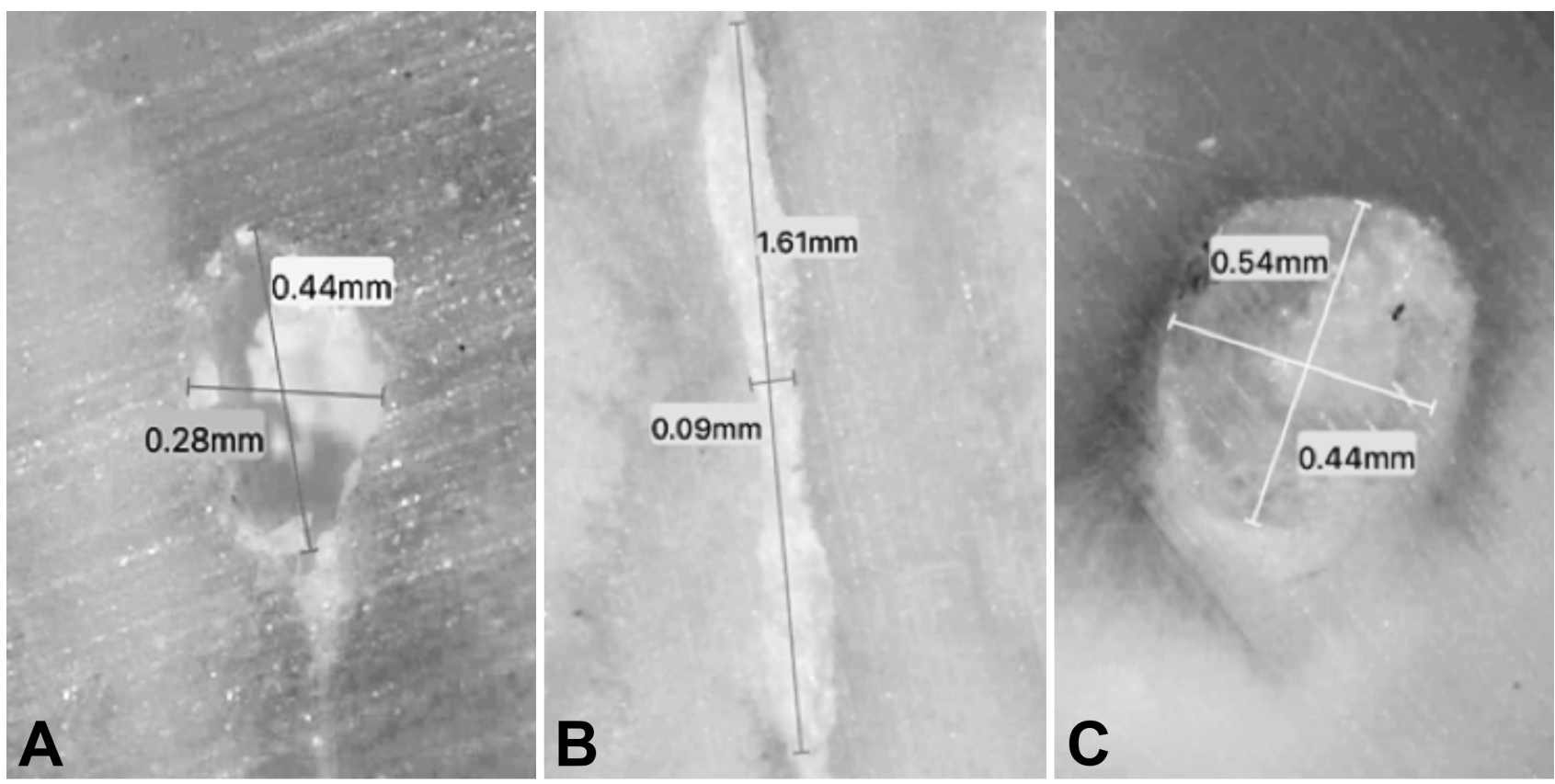

Fig. 1. Formas y mediciones apicales en distintas piezas. A) Conducto mesiobucal de primer molar superior de forma irregular con medidas cercanas a la media. B) Conducto mesial de primer molar inferior de forma ovalada con medidas alejadas de la media. C) Conducto palatino de primer molar superior de forma redonda con medidas cercanas a la media. (Microfotografías: Lilian de la Luz Aguilar Vera). 


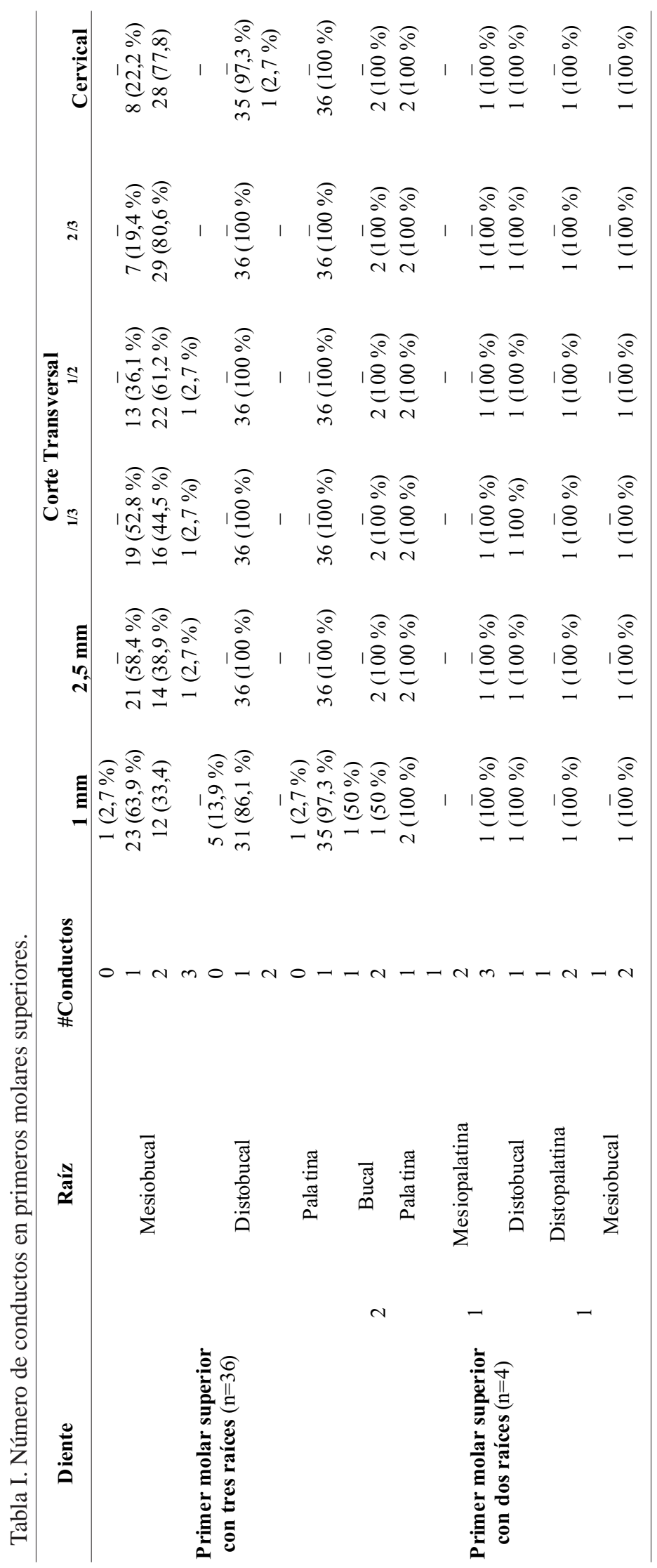

de primeros molares superiores con 3 raíces fue la configuración Tipo II (47,2\% de casos), seguida de la Tipo IV (25\%), mientras que en las raíces distobucales y palatinas la más prevalente fue la Tipo I $(97,2 \%$ y $100 \%$ de los casos). Por otro lado, en los primeros molares superiores con 2 raíces se observó un caso de raíz bucal con configuración Tipo II y otro con Tipo IV; la raíz distobucal mostró el único caso con Tipo VIII, y la raíz distopalatina reportó la Tipo IV.

Los 40 primeros molares inferiores analizados (100 \%) mostraron 2 raíces (M y D). El $30 \%$ de éstos mostraron tres conductos (MB, ML y D) desde el corte transversal cervical hasta el corte a $1 \mathrm{~mm}$ del vértice apical. El $70 \%$ de los casos mostraron variaciones en número y trayectoria de conductos radiculares (Tabla II). En las raíces mesiales, las configuraciones de Vertucci principalmente observadas fueron la Tipo II (52,5\%) y la Tipo IV (40\%), mientras que en las distales predominó la Tipo I (90\%).

Las distintas variaciones morfológicas de los conductos radiculares a $1 \mathrm{~mm}$ del vértice apical se observan en la Figura 1. La forma redonda fue la de mayor prevalencia en los conductos del primer molar superior $(44,5 \%)$, seguida de la irregular $(34,1$ $\%$ ) y la oval $(21,4 \%)$; mientras que en los primeros molares inferiores la forma más prevalente fue la irregular $(54,5 \%)$, seguida de la redonda $(23,9 \%)$ y posteriormente la oval $(21,6 \%)$.

La Tabla III muestra la distribución de formas redonda, oval e irregular en los conductos de primeros molares superiores e inferiores a $1 \mathrm{~mm}$ del vértice apical. Los conductos mesiobucales del primer molar superior y del primer molar inferior mostraron la forma irregular como la más predominante (43,6\% y $50 \%$ respectivamente). En los conductos distobucales de los primeros molares superiores predominaron las formas irregular y redonda al arrojar datos similares ( $41,2 \%$ y $38,2 \%$ respectivamente). Los conductos distales de molares inferiores tuvieron mayor prevalencia de forma irregular $(52,9 \%)$, mientras que los conductos palatinos (Fig. 1C) del primer molar superior predominó la forma redonda (61,5\% de los casos). La forma oval fue la menos prevalente en la mayoría de los casos.

Con relación a las mediciones realizadas a $1 \mathrm{~mm}$ del vértice apical; los conductos mesiobucales del primer molar superior fueron los más variables (Tabla IV; Fig. 1A), mientras que 
Tabla II. Número de conductos en primeros molares inferiores $(n=40)$.

\begin{tabular}{|c|c|c|c|c|c|c|c|}
\hline \multirow{2}{*}{ Raíz } & \multirow{2}{*}{ \#Conductos } & \multicolumn{6}{|c|}{ Corte Transversal } \\
\hline & & $1 \mathrm{~mm}$ & $2,5 \mathrm{~mm}$ & $1 / 2$ & $1 / 3$ & $2 / 3$ & Cervical \\
\hline \multirow{3}{*}{ Mesial } & 0 & $2(5 \%)$ & & & & & \\
\hline & 1 & $22(55 \%)$ & $21(5 \overline{2}, 5 \%)$ & $13(3 \overline{2}, 5 \%)$ & $6(1 \overline{5} \%)$ & $3(7,5 \%)$ & $3(7,5 \%)$ \\
\hline & 2 & $16(40 \%)$ & $19(47,5 \%)$ & $27(67,5 \%)$ & $34(85 \%)$ & $37(92,5 \%)$ & $37(92,5 \%)$ \\
\hline \multirow{3}{*}{ Distal } & 0 & $5(12,5 \%)$ & & & & & \\
\hline & 1 & $35(87,5 \%)$ & $39(9 \overline{7}, 5 \%)$ & $37(92,5 \%)$ & $34(85 \%)$ & $35(8 \overline{7}, 5 \%)$ & $36(90 \%)$ \\
\hline & 2 & _- & $1(2,5 \%)$ & $3(7,5 \%)$ & $6(15 \%)$ & $5(12,5 \%)$ & $4(10 \%)$ \\
\hline
\end{tabular}

Tabla III. Forma de los conductos de primeros molares superiores e inferiores.

\begin{tabular}{lccccc}
\hline Diente & Conducto & $\mathrm{N}$ & Redondo & Oval & Irregular \\
\hline 1er molar superior & $\mathrm{MB}$ & 39 & $13(33,4 \%)$ & $9(23 \%)$ & $17(43,6 \%)$ \\
& $2 \mathrm{MB}$ & 14 & $6(42,8 \%)$ & $4(28,6 \%)$ & $4(28,6 \%)$ \\
& $\mathrm{DB}$ & 34 & $13(38,2 \%)$ & $7(20,6 \%)$ & $14(41,2 \%)$ \\
& $\mathrm{P}$ & 39 & $24(61,5 \%)$ & $7(17,9 \%)$ & $8(20,6 \%)$ \\
Total & & 126 & $56(44,5 \%)$ & $27(21,4 \%)$ & $43(34,1 \%)$ \\
1er molar inferior & $\mathrm{M}$ & 22 & $1(4,6 \%)$ & $9(40,9 \%)$ & $12(54,5 \%)$ \\
& $\mathrm{MB}$ & 16 & $6(37,5 \%)$ & $2(12,5 \%)$ & $8(50 \%)$ \\
& $\mathrm{ML}$ & 16 & $3(18,8 \%)$ & $3(18,8 \%)$ & $10(62,4 \%)$ \\
Total & $\mathrm{D}$ & 34 & $11(32,4 \%)$ & $5(14,7 \%)$ & $18(52,9 \%)$ \\
& & 88 & $21(23,9 \%)$ & $19(21,6 \%)$ & $48(54,5 \%)$ \\
\hline
\end{tabular}

los menos variables fueron los segundos conductos mesiobucales $(2 \mathrm{MB})$ con un diámetro mayor de $0,26 \mathrm{~mm}$ y uno menor de $0,14 \mathrm{~mm}$, y desviaciones estándar de 0,08 y $0,05 \mathrm{~mm}$ respectivamente. En los molares inferiores la mayoría de los conductos mostraron considerables desviaciones de sus diámetros mayor y menor. El diámetro mayor del conducto mesial fue $0,64 \mathrm{~mm}$ y su desviación estándar fue de 0,30 mm (es decir casi $50 \%$ de su medición, Fig. 1B), mientras que el diámetro mayor del conducto mesiobucal fue de 0,42 mm y su desviación estándar de $0,29 \mathrm{~mm}$. De la misma forma, el diámetro mayor de conducto distal fue $0,54 \mathrm{~mm}$ y su desviación estándar fue de 0,24 mm (poco más del $50 \%$ de su medición). Los conductos menos variables en primeros molares inferiores fueron los mesiolinguales con desviaciones estándar cercanas al promedio de diámetros mayor y menor. Todas las mediciones apicales se observan en la Tabla IV.
Tabla IV. Diámetros mayor y menor en conductos de primeros molares superiores e inferiores a $1 \mathrm{~mm}$ del vértice apical.

\begin{tabular}{|c|c|c|c|c|c|}
\hline Diente & Conducto* & $\mathrm{N}$ & Diámetro & Promedio $(\mathrm{mm})$ & D. S. \\
\hline \multirow{8}{*}{ 1er molar superior } & \multirow{2}{*}{ MB } & \multirow{2}{*}{39} & Mayor & 0,46 & 0,29 \\
\hline & & & Menor & 0,23 & 0,09 \\
\hline & \multirow{2}{*}{$2 \mathrm{MB}$} & \multirow{2}{*}{14} & Mayor & 0,26 & 0,08 \\
\hline & & & Menor & 0,14 & 0,05 \\
\hline & \multirow{2}{*}{ DB } & \multirow{2}{*}{34} & Mayor & 0,40 & 0,17 \\
\hline & & & Menor & 0,23 & 0,08 \\
\hline & \multirow{2}{*}{$P$} & \multirow{2}{*}{39} & Mayor & 0,50 & 0,15 \\
\hline & & & Menor & 0,39 & 0,14 \\
\hline \multirow{8}{*}{$1^{\mathrm{er}}$ molar inferior } & \multirow{2}{*}{ M } & \multirow{2}{*}{22} & Mayor & 0,64 & 0,30 \\
\hline & & & Menor & 0,25 & 0,10 \\
\hline & \multirow{2}{*}{ MB } & \multirow{2}{*}{16} & Mayor & 0,42 & 0,29 \\
\hline & & & Menor & 0,21 & 0,07 \\
\hline & \multirow{2}{*}{ ML } & \multirow{2}{*}{16} & Mayor & 0,30 & 0,13 \\
\hline & & & Menor & 0,17 & 0,05 \\
\hline & \multirow{2}{*}{$\mathrm{D}$} & \multirow{2}{*}{34} & Mayor & 0,54 & 0,24 \\
\hline & & & Menor & 0,33 & 0,14 \\
\hline
\end{tabular}

*MB= Mesiobucal, $2 \mathrm{MB}=$ segundo Mesiobucal, $\mathrm{DB}=$ Distobucal, $\mathrm{P}=$ Palatino, $\mathrm{M}=\mathrm{Mesial}$, $\mathrm{ML}=$ Mesiolingual, D=Distal. D.S. Desviación Stándar.

\section{DISCUSIÓN}

El presente estudió encontró diferencias con respecto a otros estudios en el número de raíces tanto en el primer molar superior como en el molar inferior. Kerekes \& Tronstad (1977) reportaron en una población caucásica una prevalencia de primeros molares superiores con tres raíces del $65 \%$ y del $20 \%$ con dos, mientras que el presente estudio obtuvo un $90 \%$ de primeros molares con 3 raíces y sólo un $10 \%$ mostró dos.

Con relación a los primeros molares inferiores, Drennan et al. (1929) reportaron datos semejantes a lo encontrado en este estudio al describir el $100 \%$ de sus casos con 2 raíces en una poblacón sudafricana, mientras 
que Sperber \& Moreau (1998) registraron un 96,9\% con 2 raíces y un $3,1 \%$ con 3 raíces en una población africana. Por otro lado, Gulabivala et al. (2001) reportaron un $90 \%$ con 2 raíces y un $10 \%$ con tres; Huang et al. (2010) documentaron frecuencias más discrepantes en una población de Taiwán donde el 74,7 \% tuvo 2 raíces (mesial y distal) y el 25,3\% fue de 3 raíces ( 2 mesiales y una distal).

El sistema de conductos del primer molar superior según la clasificación de Vertucci (1984) mostró la configuración Tipo I como la más prevalente en las raíces distobucales y palatinas del presente estudio y la Tipo II en las raíces mesiobucales. Martins et al. (2018) reportaron datos similares en una población caucásica (Portugal), arrojando una mayor prevalencia de Tipo I en las raíces distobucales y palatinas $(89,2 \%$ y $89,4 \%$ respectivamente) y el Tipo II $(40,6 \%)$ en las raíces mesiobucales. Por otro lado, autores como Lee $e t$ al. (2011) y Weine et al. (1999) mostraron mayor prevalencia de configuración Tipo IV (34,9\%) y Tipo I (42\%) en poblaciones koreanas y japonesas respectivamente.

En primeros molares inferiores Gulavibala et al. (2001) reportaron en una población burmés una mayoría del Tipo I $(38,1 \%)$ para las raíces mesiales, mientras que el presente estudio arrojó una mayor prevalencia de configuración Tipo II $(52,5 \%)$ en la misma raíz. No obstante, en las raíces distales hubo similitud entre Gulavibala et al. y el presente, donde la configuración de mayor prevalencia fue la Tipo I (80\% y $90 \%$ respectivamente). Martins et al. reportaron similitudes con respecto al presente estudio en ambas raíces de primeros molares inferiores: la raíz mesial tuvo su mayor prevalencia de configuración Tipo II, mientras que la raíz distal fue el Tipo I.

De forma general, la morfología de los conductos radiculares a $1 \mathrm{~mm}$ del vértice apical en primeros molares superiores e inferiores reportó para la población yucateca diferencias con respecto a otras poblaciones dentro del continente americano. Abarca et al. reportaron una mayor prevalencia de forámenes fisiológicos con forma oval ( $50 \%$ ) en los conductos del primer molar superior, seguida de la forma irregular $(32 \%)$ y la forma redonda $(18 \%)$ en una población chilena. El presente estudio reportó mayoría de conductos redondos $(44,5 \%)$, seguido de la forma irregular $(34,2 \%)$ y por último la forma oval $(21,4 \%)$. De forma semejante, Gani \& Visvisian encontraron a $2 \mathrm{~mm}$ del vértice apical, la configuración oval como la más prevalente en los conductos mesiobucales del primer molar superior $(32,5 \%)$, seguida de la forma redonda $(15 \%)$ en una población americana caucásica; mientras que Marroquín et al. (2004) y la presente investigación reportaron mayor prevalencia de configuración irregular (74,6\% y 43,6\% respectivamente) en los mismos conductos. En los conductos distobucales del presente estu- dio predominó la forma irregular, de manera semejante a lo encontrado por Marroquín et al., pero de forma contraria a lo reportado por Gani \& Visvisian quienes obtuvieron una mayor prevalencia de forma redonda $(45 \%)$.

Por último, cabe mencionar que, en los conductos mesial y distal del primer molar inferior, la mayoría de los autores mencionan como la forma más prevalente la configuración irregular, con excepción de Abarca et al., quienes reportaron en forámenes fisiológicos la forma oval como la de mayor índice (59\%), seguida de la irregular $(23 \%)$ y la redonda (18\%). Lo anterior difiere de los resultados encontrados en esta investigación, donde la forma con mayor prevalencia fue la irregular $(54,5 \%)$, seguida de la redonda $(23,9$ $\%)$ y, por último, la oval $(21,6 \%)$.

Las medidas de los diámetros mayor y menor de los conductos radiculares a $1 \mathrm{~mm}$ del vértice han sido un punto de referencia para la instrumentación en las distintas poblaciones; así Wu et al. (2000) reportó que en una población de Ámsterdam (Países Bajos), los primeros y segundos molares mostraron un conducto mesiobucal con medidas prácticamente similares a las encontradas en el presente estudio con un diámetro mayor de $0,43 \mathrm{~mm}$ y uno menor de $0,22 \mathrm{~mm}$, mientras que nuestro estudio arrojó un diámetro mayor de $0,46 \mathrm{~mm}$ y uno menor de $0,23 \mathrm{~mm}$. Sin embargo, las mediciones de los demás conductos no concuerdan con el presente estudio; el segundo conducto mesiobucal del presente estudio arrojó un diámetro mayor de $0,24 \mathrm{~mm}$ y uno menor de $0,14 \mathrm{~mm}$, mientras que Wu et al. reportaron un diámetro mayor de $0,19 \mathrm{~mm}$ y uno menor de $0,16 \mathrm{~mm}$ para el mismo. En los conductos distobucales, Abarca et al. describieron para forámenes fisiológicos un diámetro mayor de $0,32 \mathrm{~mm}$ y uno menor de 0,25 $\mathrm{mm}$, mientras que Wu et al. reportaron un diámetro mayor de $0,22 \mathrm{y}$ uno menor de $0,17 \mathrm{~mm}$. Ambas medidas fueron menores que lo reportado en el presente estudio, donde se observó un diámetro mayor de $0.40 \mathrm{~mm}$ y uno menor de $0,23 \mathrm{~mm}$ para los conductos distobucales. De igual forma, los conductos palatinos Abarca et al. y de Wu et al. reportaron mediciones de diámetros mayor y menor alrededor de $0,36 \mathrm{~mm}$ y 0,28 $\mathrm{mm}$ respectivamente, mientras que la media para dichos conductos en esta investigación fueron superiores (diámetro mayor $=0,50 \mathrm{~mm}$, diámetro menor $=0,39 \mathrm{~mm}$ ).

Con lo que respecta a los primeros molares inferiores, las diferencias más notables fueron con respecto a $\mathrm{Wu}$ et al. (2001), quien reportó un diámetro mayor de $0,45 \mathrm{~mm}$ y uno menor de $0,22 \mathrm{~mm}$ para el conducto mesial, mientras que el presente estudio obtuvo medidas mayores (diámetro mayor $0,64 \mathrm{~mm}$ y diámetro menor de $0,25 \mathrm{~mm}$ ). Los conductos mesiolinguales y distales de Wu et al. (2001) también reportaron medidas discrepantes a lo reportado en el presente estudio, y únicamente los conductos mesiobucales mostraron 
similitudes. El diámetro mayor de conductos mesiobucales de Wu et al. (2001) fue de 0,40 $\mathrm{mm}$ y el menor de $0,21 \mathrm{~mm}$, el presente estudio reportó un diámetro mayor de $0,42 \mathrm{~mm}$ y uno menor de $0,21 \mathrm{~mm}$ también para los mismos conductos.

\section{AGRADECIMIENTOS}

Agradecemos a Fernando Aguilar Ayala, Ruben Herrera Atoche y Mauricio Escoffié Ramirez, director y autoridades de la Facultad de Odontología de la UADY, por las facilidades brindadas para la realización de este trabajo. También a la Secretaría de Educación Pública a través del programa de mejoramiento de profesorado (POMEP) UADY-PTC-225 a cargo de E.M.V.L.; Proyecto CONACYTCV-152105 de V.T. y el Proyecto CONACYT-CV-154750 de A.C. que proporcionaron financiamiento. Los autores declaran no tener conflicto de intereses con relación al presente texto.

VEGA-LIZAMA, E. M.; AGUILAR-VERA, L. L.; TIESLER, V.; RAMÍREZ-SALOMÓN, M.; ALVARADO-CÁRDENAS, G.; LÓPEZ-VILLANUEVA, M. E. \& CUCINA, A. Root morphology and apical measurements of first molars in a Mayan population. Int. J. Morphol., 36(4):1229-1234, 2018.

SUMMARY: Accurate knowledge of dental root morphology is a key to success in endodontic therapies. The aim of this study was to evaluate the different variations of the root and apical morphology in upper and lower first molars of a modern Mayan population. Photomicrographs were taken from 80 upper and lower first molars, presented in cross sections at 1 and $2.5 \mathrm{~mm}$ from the apical vertex, as well as from the $1 / 3,1 / 2,2 / 3$ portions of the root length and at the cementoenamel junction. The number of roots, canal and Vertucci`s configuration was determined, as well as the shape and measurements of the major and minor diameters at $1 \mathrm{~mm}$ from the apex. In this study $90 \%$ of upper first molars reported the presence of 3 roots and $10 \%$ showed 2, while the total lower first molars $(100 \%)$ reported 2 roots. In general, Vertucci's Type II prevailed in the mesial roots while Type I was prevalent in the distal and palatal ones. In the $1 \mathrm{~mm}$ cut of the apical vertex, the round shape was the most prevalent in the canal of the first upper molars (44.5\%), followed by irregular (34.1\%) and oval $(21.4 \%)$; while in the first lower molars the most prevalent one was irregular $(54.5 \%)$, followed by round (23.9 $\%)$ and oval $(21.6 \%)$. The major and minor diameters of the mesial canals at $1 \mathrm{~mm}$ apical recorded 0.46 and $0.23 \mathrm{~mm}$ and 0.64 and $0.25 \mathrm{~mm}$ in upper and lower molars respectively. The frequency of MB2 in upper first molars was $77.8 \%$. The canals with greater variability were the mesial ones. Low frequency of oval canals was observed. The largest diameters of the mesial canal in general were greater than $0.45 \mathrm{~mm}$.

KEY WORDS: Root anatomy; Root canal; First molar; Apical diameter.

\section{REFERENCIAS BIBLIOGRÁFICAS}

Abarca, J.; Zaror, C.; Monardes, H.; Hermosilla, V.; Muñoz, C. \& Cantin, M. Morphology of the physiological apical foramen in maxillary and mandibular first molars. Int. J. Morphol., 32(2):671-7, 2014.

de Pablo, O. V.; Estevez, R.; Péix Sánchez, M.; Heilborn, C. \& Cohenca, N. Root anatomy and canal configuration of the permanent mandibular first molar: a systematic review. J. Endod., 36(12):1919-31, 2010.

Drennan, M. R. Dentition of the Bushman tribe. Ann. S. Afr. Mus., 24:61-87, 1929.

Gani, O. \& Visvisian, C. Apical canal diameter in the first upper molar at various ages. J. Endod., 25(10):689-91, 1999.

Gulavibala, K.; Aung, T. H.; Alavi, A. \& Ng, Y. L. Root and canal morphology of Burmese mandibular molars. Int. Endod. J., 34(5):359-70, 2001

Hargreaves, K. M. \& Berman, L. H. Cohen's Pathways of the Pulp. $11^{\text {th }}$ ed. St. Louis, Elsevier, 2016.

Huang, C. C.; Chang, Y. C.; Chuang, M. C.; Lai, T. M.; Lai, J. Y.; Lee, B. S. \& Lin, C. P. Evaluation of root and canal systems of mandibular first molars in Taiwanese individuals using cone-beam computed tomography. J. Formos. Med. Assoc., 109(4):303-8, 2010

Kerekes, K. \& Tronstad, L. Sweden, M. Morphometric observations on the root canals of human molars. J. Endod., 3(3):114-8, 1977.

Lee, J. H.; Kim, K. D.; Lee, J. K.; Park, W.; Jeong, J. S.; Lee, Y.; Gu, Y.; Chang, S. W.; Son, W. J.; Lee, W. C.; Baek, S. H.; Bae, K. S. \& Kum, K. Y. Mesiobuccal root canal anatomy of Korean maxillary first and second molars by cone-beam computed tomography. Oral Surg. Oral Med. Oral Pathol. Oral Radiol. Endod., 111(6):785-91, 2011.

Marroquín, B. B.; El-Sayed, M. A. \& Willershausen-Zönnchen, B. Morphology of the physiological foramen: I. Maxillary and mandibular molars. J. Endod., 30(5):321-8, 2004

Martins, J. N. R.; Ordinola-Zapata, R.; Marques, D.; Francisco, H. \& Caramês, J. Differences in root canal system configuration in human permanent teeth within different age groups. Int. Endod. J., 51(8):931-41, 2018.

Oliva Rodríguez, R.; Gastélum Zazueta, A. G.; Hernández Molinar, Y.; Gutiérrez Cantú, F. J. \& Silva-Herzog Flores, D. Incidence and type of isthmus in human permanent first molars, in vitro assessment. Int. J. Morphol., 35(4):1280-4, 2017.

Sperber, J. H. \& Moreau, J. L. Study of the number of roots and canals in Senegalese first permanent mandibular molars. Int. Endod. J., 31(2):17722, 1998.

Vertucci, F. J. Root canal anatomy of the human permanent teeth. Oral Surg. Oral Med. Oral Pathol., 58(5):589-99, 1984.

Weine, F. S.; Hayami, S.; Hata, G. \& Toda, T. Canal configuration of the mesiobuccal root of the maxillary first molar of a Japanese sub-population. Int. Endod. J., 32(2):79-87, 1999.

Wu, M. K.; R'oris, A.; Barkis, D. \& Wesselink, P. R. Prevalence and extent of long oval canals in the apical third. Oral Surg. Oral Med. Oral Pathol. Oral Radiol. Endod., 89(6):739-43, 2000.

Dirección para correspondencia:

Elma María Vega-Lizama

Dirección Calle 44A No. 232 por 23 y 25, Col.

Roma, C.P. 97218

Mérida

Yucatán

MÉXICO

Email: elma.vega@correo.uady.mx 